Article

\title{
The Violation Imperative Part 1: Allegations \& Fraud
}

\section{La Shun L. Carroll ${ }^{1}$}

1 The University at Buffalo Graduate School of Education 1; 1carroll@buffalo.edu

\begin{abstract}
In recent decades, a number of high-profile cases involving fraud as research misconduct have been in the media and resulted in severe consequences for those convicted. According to the increased cases of allegations and coverage in the media, this reflects a heightened awareness that fraudulent actions exist. Nonetheless, the Office of Research Integrity data suggests that despite the growth in the number of the cases of allegation there has not been a commensurate increase in findings of misconduct. The purpose of this paper is to explore misconduct to better understand what it entails. An analysis of misconduct from the perspective of the definitions of allegations and fraud of is conducted and potential frameworks for understanding both are considered. The paper considers serial-positioning effects of primacy and recency on allegation phenomena, as well as supervenience theory and contextualism as a lens for understanding fraud. Discussion of the relational semantics of the core aspects of fraud and de facto grouping of forms of misconduct. It is concluded that the interrogative pronouns of "what" and "when" could be used to categorize forms of misconduct laying the foundation for the next paper that deconstructs the definition of falsification according to the Public Health Service.
\end{abstract}

Keywords: Fraud, Allegations, Ethics, Research Misconduct, Philosophy

\section{Introduction}

According to data from case summaries of misconduct outcomes between 2006 and 2015 from the Office of Research Integrity (ORI), while the number of cases of misconduct being considered has increased, the number of cases in which there were findings of misconduct has remained relatively consistent [1]. This trend has the disturbing corollary that not only has made false allegations more frequent, but because of the increased number of accusations despite the absence of a commensurate increase in findings of misconduct, more individuals are falsely accused. Moreover, such an increase in false accusations implies that, unless only those guilty of misconduct are the ones whose cases result in findings of guilt, which is untrue, there are necessary increases in the number of false convictions as well tarnishing personal and professional reputations - sometimes, irreparably.

Regardless the social, ethical, or legal transgression of which one may be accused, one thing is sure: there will always be a proportion of such allegations that ultimately and inevitably will be determined false. Nevertheless, the certainty of occurrence in no way implies the frequency of such. Even given the most generous estimate of frequency, it would be difficult to imagine -let alone accept - that false allegations are being made nearly as often as instances in which there is a legitimate basis for whistleblowing. Unfortunately, however, between 2006 and 2015, for each year except just one outlier, more cases were resulting in no findings of misconduct than there were those in which there were findings. Furthermore, in the absence of any significant increase in actual findings of research misconduct occurring, any attempts at understanding the data become rather complicated.

Efforts to reconcile the trends in the data and possible justifications for them were the impetus behind this paper. Could all of the accusers responsible for the false allegations during all but one year outnumber legitimate complainants? If so, what motivated the false allegations? Could they have hallucinated yet truly believed? Perhaps the accusers were fully aware of their actions intentionally making false allegations? Better still, although it is not high on a tentative list of explanations, in all fairness maybe the problem is not related to the whistleblowers at all. Unless year after year there is evidence to support the same accusers making allegations that cannot be 
substantiated based on there being any finding of misconduct in cases, which is very unlikely, there is only one other commonality that potentially connects the cases. This commonality does not relate to the motive, intent, or action of the accusers at all; it concerns the gatekeepers in charge of determining whether to hear the cases and rendering decisions concerning cases heard.

Although due diligence requires that all realistic possibilities be given serious consideration, jumping to the absolute worst conclusion would mean that either the people were making more false allegations, or that those sworn to uphold the virtues of the profession and the entire scientific community at large have orchestrated some fantastically brilliant but devious plan conspiring to limit findings in cases heard in order to bolster the image of adequate self-regulatory capacity. It would be more reasonable that the problem lies not with actions, or the failure to act. Giving people the benefit of the doubt that they are genuinely doing their best to maintain integrity, the issue that would be consistent with adopting this framework for viewing the phenomena of false allegations would be the presence of a flaw or defect with the manner in which the classifications comprising misconduct are defined. Such a flaw contributes to misunderstanding what constitutes an instance of misconduct.

Depending on one's perspective, both the best and the worst aspect of allegations, in general, is the same: allegations do not require evidence. This aspect is consistent with the fact that, if there were to be evidence of what is alleged, then there could not be any allegations; there would be findings of the transgression in question. It is only after the ruling of no findings that allegations may be determined false. Unfortunately, by the time a ruling of no findings has been made, the damaging effects have already occurred to the individuals who have been cleared of wrongdoing.

While it may be the case that transgressions - be they criminal, ethical, or otherwise - do not always leave evidence of their occurrence, does this imply they ought not to be required to make allegations? Without evidence, on what ground would allegations rest? Before we can consider this and other questions, we must understand what it means to "allege."

\subsection{Denotative Meaning of Allege}

By definition, to allege is "to assert without or before proof [2] (Merriam-Webster 2017)." On the one hand, individuals wrongly accused of having committed misconduct may view the absence of an evidence requirement in order to make allegations as the worst possible thing. Since there is no evidence required to make a claim against others, it does not matter whether one will ultimately be exonerated because nothing will have changed concerning the status of evidence. Nevertheless, at best, he or she will always be remembered for having been rightfully cleared of allegations of the misconduct of which they were accused.

Alternatively, at worst, he or she may potentially be wrongly convicted for something that he or she did not do, which occurs often enough to merit the creation of scholarly works dedicated entirely to the phenomenon of False Convictions concerning various types of crime and misconduct [3] (Turvey, Savino, \& Baeza, 2017). Regardless of which outcome occurs, neither exoneration nor false conviction is particularly beneficial or designed to protect those unjustly accused. Although there are procedures in place to appeal when falsely convicted, there are no such means in the case of those falsely accused. In particular, even when exonerated of alleged misconduct after being falsely accused, one is genuinely never wholly "cleared" in the eye of the many due to flawed reasoning that leads to the erroneous belief that only the guilty are alleged to have committed transgressions. This phenomenon of persistent guilt despite the lack of findings is reminiscent of the manner in which allegation phenomena operate. Because of their similarities, we will begin by considering a potential framework for perceiving allegation phenomena and what may be responsible guilt persistence despite the absence of evidence or reason.

\subsection{Serial-Positioning as a Framework for Perceiving Allegation Phenomena}

With such a poor outlook for anyone falsely accused regardless of the outcome of their hearing, the phenomenon of allegations and their aftermath merit consideration in the author's opinion. As a potential tool for analysis borrowed from a different field, the author proposes that observations be 
understood from the perspective of the effects of serial-positioning (i.e., presentation order of items) on recall [4] (Neiman, Loewenstein, \& Shteingart, 2013).

Concerning primacy versus recency, as it relates to serial-positioning, it has been observed that the last or most recent item in a list of items, is what people are most likely to freely recall while secondmost-likely in a list of items studied for subsequent recall are those that were among the first encountered. It is based on such research findings that in textbooks on academic writing suggest structuring the series of points for one's argument with the second-most important point mentioned initially and the most critical point stated last [5] (Behrens \& Rosen, 2018). Applied as a lens through which to view the impact of allegation, primacy guiding cognitive bias in the best and worst scenarios would predict allegations themselves standing out regardless of any subsequent findings to be most likely recalled whereas exoneration either at trial initially or upon appeal subsequently occurring lastly being recalled would implicate recency effects as the dominating force behind cognitive bias.

Given the damaging effects of allegations to the personal and professional lives of individuals accused even when allegations are immediately discredited, or discrediting is delayed, primacy is that which appears to take precedence. However, despite this apparent primacy effect, the basis for it is both unintuitive and unclear. Moreover, in the face of multiple allegations on separate occasions, the primary effect seems to be reinforced.

What is interesting is that over time, the primacy effect is transient. Transience makes sense when considering that the longer a list of items may be, the longer the time since the first item was mentioned, which affects recall. Nonetheless, it is recency that not only determines what is most likely to be recalled, but has also been shown to increase recall with successive trials [6] (Huang, Tomasini, \& Nikl, 1977). Thus, recency would be expected to guide recall in the case of exoneration of the innocents, which means the outcome of allegations and not the allegations themselves are what should be recalled. Persistence of the presumption of guilt based on having been alleged despite the exoneration is very real. The question is why this is the case?

One explanation is that there may be a crucial difference between a list of items for recall and actual events such as allegations: the inextricable link to, and attribution of, values. That is a series of letters, or unrelated words one is asked to study for later recall would be devoid of any meaningful, inherent attributes that rely on judgment or assessment. Conversely, concerning a list comprising a string of events that one is instructed to study for later recall in which there is a piece of information included stating a prominent scientist is alleged to have committed research misconduct, appended to the content of the item itself would be some form of valuation resulting in the attribution of either good, bad, or possibly neutral. In this particular instance, the information content would likely be labeled with some variety of semantic tagging much the same way an internet framework called "CogTag" has suggested [7] (Wu, Qian, Riguidel, \& Chen, 2013). When later prompted for free recall, undoubtedly certain tagged items (e.g., deemed good or bad) would come to mind before others.

Given the incessant war waged between our evolutionary pressures to the self and those to the group [8], it would make sense that events that are labeled and interpreted as threats are more readily recalled. Furthermore, of the two evolutionary pressures, the pressures to the self are given priority over those to the group since self-preservation is the best way to guarantee one's ability to satisfy both pressures. Attentional resources are quite supple and may be directed according to the needs of the particular task of the moment [9], and the task above all else is self-preservation, the ability to direct one's attention as required with the tagged "bad" items in memory could explain the observed behavior of persistence of guilt and the aftermath of false allegations in general. On a primal level, recalling or remembering people for being targeted with allegations despite them being ultimately dismissed may be due to being perceived as potential red flags and threats to the group, therefore, the self.

\subsection{Allegations and Circular Reasoning}

From the perspective of the one making allegations, the absence of an evidence requirement may be perceived as the best thing about alleging because it places the onus effectively on the accused and the "system." Nonetheless, if someone were to be wrongfully accused but fortunate enough to be 
exculpated due to lack of evidence found, then things would be as they should. If no evidence of research misconduct was required for the allegations, however, then there should be no surprise if none were ever to be found.

Whether evidence ought to be required, more important to address is what constitutes evidence. How evidence is understood as it pertains to research misconduct, especially by accusers, depends as much on the meaning of the definition to people as it does on how they interpret the definition. According to a very commonly used definition of research misconduct given by the Public Health Service (PHS), my attempt to understand the definition of falsification as research misconduct was the least successful. The cause of the difficulty was due to the circular logic, which was used to construct it.

While nothing can completely eliminate the problems understanding even once a definition is adequately addressed, given that there always exists the potential for something so devastating to occur to anyone, success in small increments through refinement of definitions or criteria that this work aims to accomplish is what ultimately contributes to overall progress. Furthermore, that there exists a possibility of being falsely accused should be cause for concern regarding allegations in general, as well as those related to research misconduct in particular because there is a sizable cost associated with being involved regardless of the outcome of the allegations.

\subsection{The Aggregate Cost of Allegations}

For obvious reasons, it should be understood that the costs associated with all allegations of research misconduct may be sufficient to financially strain one personally and professionally. Moreover, regardless of whether truly guilty of misconduct, when the outcome of allegations is not in one's favor, this may result in severe consequences.

In addition to being costly, to go from an allegation to a ruling in a suspected case of research misconduct is a protracted process from beginning to end. Research regarding cost estimates attempted to determine the aggregate cost (AC) of a case of scientific fraud using a statistical method that incorporates both measurable and intangible costs [10] (Michalek et al. 2010). The AC estimate produced from the analysis conducted by Michalek et al. [11] 2010 was calculated to be $\$ 525,000$.

Costs and lengthy hearings associated with cases are not the only setbacks resulting from allegations. According to work done by Hey \& Chalmers [12] (2010), which discusses how allegations of suspected misconduct that were unfounded led to a case that was widely publicized when it occurred in the UK, allegations may be responsible for damage to defendants lives even when no evidence is ever found. It was the resultant damage that still occurred despite the absence of proof that is most troubling. The damage occurred throughout the eleven years it took to determine that there was no real basis for the allegations made.

The ultimate cost of this UK case was more than an estimated $£ 6$ million in total [12] (Hey and Chalmers 2010). Given the repercussions of allegations, if possible, more ought to be done to improve the process. The most problematic aspect from any defendant's perspective is that the sole recourse that the accused may have if another alleges misconduct is to attempt to sue for libel [12] (Hey and Chalmers 2010). However, to successfully sue for defamation as a strategic legal maneuver may prove exceedingly difficult.

The difficulty would be in proving how allegations may be considered libelous, or defamatory given the possible outcomes. On the one hand, if the accused is convicted on the basis of findings produced during a trial, then no "good character" can be said to have been tarnished because there exists evidence, which nullifies the potential for libel claims. Conversely, if acquitted, the exoneration of the accused is supposed to ensure that his or her reputation remains intact. Unfortunately, as mentioned previously, this is not how it tends to be for the accused whether exculpated or not.

It appears as though one may become easily ensnared by the web of allegations of research misconduct. The facilitation originates from the lack of evidence required. That notwithstanding, allegations are seemingly impossible to escape. In fact, the reason they are seemingly impossible to truly escape is the very same one that makes them seem as impossible to avoid. 
Although the absence of evidence may eventually result in the case being found not to have merit, due to the very same lack of proof required to allege, a conviction is not needed for damage to occur. While combating damage may be exceedingly difficult ex post facto, to target allegations directly as a preventive measure is within reason. Thus, the approach that I will use consists of addressing the definition and aspects of what is alleged: fraud as a type of research misconduct. Once fraud has been considered and a determination made concerning the nature of it broadly, the next paper will focus on the definition of falsification as a type of fraud concerning research misconduct as it is defined in a widely accepted established definition of the Public Health Service (PHS).

Because the entirety of research misconduct may not be adequately addressed in any single article, as part one of a multi-part philosophical critique, I will consider only a single pillar that comprises the definition of research misconduct. By concentrating on one central aspect in each paper, the total result of my work in this series may provide understanding and allow for the reframing of suspected instances of research misconduct. Also, any novel ideas that are derived from the findings may have the potential to contribute to discourse concerning the ethical conduct of research and possibly prevent at best, or reduce at least, future allegations.

\section{Geometrical Method of Deconstructing Falsification as Fraud Under Research Misconduct}

Fabrication, falsification, and plagiarism (FFP) that comprise explicit definitions of research misconduct may each be considered to result in instances of fraud when these acts are perpetrated. As an explicit definition, the definition of falsification provides sufficient detail to allow for identified instances matching the definiens (i.e., the description) to be substituted with the definiendum (i.e., word) "falsification" being defined [13] (Cook 2009). Insofar as the term is routinely understood, fraud may be defined as "that which is done with the intent to deceive" [14] (Fellbaum 1998). According to this definition of fraud, one may infer that there exist at least two aspects:

1. The aspect of intention and

2. The aspect of deception.

What is interesting to note is that, despite their existence, neither the aspect of intent nor that of deception may be directly observable or measured, which renders them both theoretical constructs [15] (Martella, 2013). As mental abstractions, theoretical constructs are often relied upon to convey ideas, concepts, or notions that defy immediate perception. Furthermore, any percept comprised of an instance of a construct shares underlying characteristics with all others. It is only through the realization of such percepts that one is justified in claiming the existence of constructs.

A claim of understanding concerning fraud in this capacity would be that the core aspect of fraud is that of intent. Core aspect refers to that which is requisite, foundational to the occurrence of fraud, or considered that without which it would be impossible for fraud to occur. That is, while deception can occur without intent, as we will discuss in the remainder of this section, the absence of intention would violate the definition given, as well as most commonsense understandings of fraud. That notwithstanding, although the core aspect of intent may be necessary, it must still be qualified by the aspect of deception.

Qualification of intent by the aspect of deception influences what counts as fraud in the following two significant ways. Firstly, qualification affects the aspect of intent by establishing a relationship that resembles equivalence superficially. Such a resemblance whereby intent is interpreted to be the deception leads to an explicit relationship in which one may be thought of as being interchangeable with the other. Nonetheless, the substitution of one for the other is, in fact, not possible. Unfortunately, "intent" cannot be substituted by "deception" in any place that it occurs because each instance of deception does not necessarily have to correlate with that of intent. The qualification of intent by deception may be best understood as descriptive. As such, qualifiying intent with deception serves only to explain how things were, are, or will be [16] (Browne and Keeley, 2015).

The second way in which the qualification functions is in its subordination of the aspect of intent itself. By subordination, I refer to a reduction in the status of the aspect of intent to that of deception. A reduction occurs in which the property of being perceptible becomes required for the aspect to be realized. 
As counter-intuitive as it may seem, I view the additional property of being perceptible as a reduction in status because it results in another requirement. For instance, both intent and deception can exist independently of one another. However, it is only through specific acts of deception (e.g., the example of check forgery) that intention may be inferred. In this respect, it is impossible for intention to be perceived directly; thus, it may only be so indirectly. Conversely, deception may be perceived by both direct and indirect means. Going from direct to indirect perception is the reduction of intention to which subordination refers.

The reduction of the status of intent to that of deception is due to the absence of true bidirectionality, or biconditionality. Bicondionality, as a class of relation between two objects, refers to the symmetry of conditionalization such that if A then B, then if B then A [13] (Cook 2009). From the truth of such symmetry, it may be understood why biconditionality is used to represent or describe one concept of equivalence [13] (Cook 2009). Equivalence among the two aspects of intent and deception that results from a reduction in status of intent is established through the construct of subordination.

The aspect of intent is conceptual and immaterial whereas that of deception manifests itself in observable forms. Given the nature of, and the relation between, both intent and deception, deception is directly verifiable whereas intent may only be confirmed through a performative behavior, such as an act of deception. Therefore, despite rendering the aspect of intent less powerful, the reduction of intent to the level of deception through subordination serves the purpose of making it possible for intent to be verifiable.

\subsection{Relational Semantics of Intent and Deception}

The set of aspects comprising intent and deception, as qualities or properties, are related to one another in a variety of ways. A property or quality may be understood to refer to that which may be attributed to something or predicated of that thing [17] (Orilia \& Swoyer, 2017). Individually, considering each of the relations that one aspect bears to the other, or to itself alone, renders the relation to be binary. By exploration of some of the binary relationships among the properties in an example case of fraud, we may gain insight that guides the process of interpretation. For this reason, a hypothetical case concerning fraud involving cashing a forged check follows for consideration, which should lead to a more profound understanding of both intent and deception.

Let us begin by supposing that there actually exists a forged check and that the forged check was successfully cashed. From the actual existence of this forged check, it will be our goal to determine, concerning the two aspects of intent and deception, as much as possible regarding their relationship, implications, and entailments.

Forgery may be defined as the act of producing a copy of either a document, signature, or work of art [18](Oxford 2017a). Given that something is copied, through this definition one may interpret forgery in the context of an absence of authenticity. By virtue of both the behavior of the individual and the act itself associated with the committal of forgery, outside of coercion, entrapment or otherwise being forced against one's will, it can be inferred that the person responsible for committing fraud could only have intended for deception to occur.

By copying the signature of another person onto the check, going to the bank, and handing the forged check over, it may be concluded that passing his or her signature off as that of another so as to cash the check was not only their intention, but upon successful completion was also to become their deception. That notwithstanding, intending for deception to occur does not imply that deception will result. Moreover, the concept of forgery has multiple frameworks from which to be interpreted as fraud.

The frameworks we will consider include the perspective of the one committing the act (i.e., intention or first-person), the one not committing it (i.e., deception or second-person), from within the framework of the process itself, which is active (i.e., copying the signature of another) and from that of the product that results (i.e., consequences of the act). We must ask whether the existence of multiple perspectives in any way influences what would be categorized as fraud? If so, then should it and for what reason. The answers provided to these types of question-prompts as thought 
experiments will reveal much about what we perceive and how we do so. For instance, considering fraud from a framework of intention alone regardless whether there is a successful outcome, from the standpoint of success in deception regardless of the existence of intent, from that of the action itself regardless of the intent or success, or from the consequences regardless of the action, is there an example that might qualify as an instance from within all four frameworks? A reevaluation of what characterizes fraud is required.

\subsection{Thought Experiment: Characterizing Fraud}

We have thus far assumed that intent to deceive is requisite for fraud to occur. Nonetheless, I upon reconsideration of the manner in which fraud is characterized from various perspectives, I contend that intent is neither core nor is it even pertinent to the process of committing fraud. I will discuss a hypothetical case concerning a forged check and demonstrate how the definition of fraud as it currently stands may be flawed. At each step, it will be the goal to challenge our assumptions by attempting to find a counterexample or an exception.

Let us suppose that there is a check that was signed for and cashed by a person for whom it was not meant. The person who wrote the signature for the check, therefore, must have intended to deceive. Unless a counterexample can be thought of, upon successfully cashing the check, he or she has committed fraud. To best characterize what has transpired, it is either the case that this forger had to have signed the name of another, or he or she just had to have not signed his or her name. Which is an adequate phrasing to characterize this? In other words, concerning fraud, is it more important to propose that the forger signed the name of another person, or that he or she did not put his or her own signed name down?

In order to decide which wording should be used to frame the act of forgery, one needs first to determine whether it is possible for both versions of the criterion to be consistent with one another. By consistent, it is meant that both versions of the framing of criteria may be correct simultaneously without contradiction. An example analogous to this may be found in the Principles of Ethics in which comparisons are made between beneficence and nonmaleficence.

\subsection{Beneficence Versus Nonmaleficence}

When comparing doing good by beneficence with not doing harm through nonmaleficence, one is afforded the opportunity to potentially see the same thing from multiple perspectives. Nonetheless, while it may be true that doing good would be considered not doing harm, not doing harm is not necessarily considered the same as doing good, which demonstrates the necessity in having the perspectives of the same thing from both as principles.

The key to comparing multiple perspectives of the same phenomenon is the construction of both an affirmation and corresponding denial statement. For instance, concerning the notion of good, constructing it as a principle of ethics in the form of "doing good" (i.e., beneficence) initiates the comparison through the use of a verb. After the verb-phrase, the next stage requires the construction of the negated form, or "not" + verb. However, we are left with "not-doing good" and not doing good is not equivalently formulated. Since this negated construction as opposite cannot be compared because it is not the same, it is corrected by extending the negation to the notion of good itself (e.g., think double negation). In this fashion, once "not good" is obtained, it may be substituted for its equivalent (i.e., harm) to result in "not doing harm."

The reasoning for the suggestion mentioned above is to allow for the analysis of the general structure of the formulae. I conceived of this procedure as being analogous to what occurs in higherorder logic in which a particular realization is generalized in order to evaluate its logical structure. For instance, if "John writes" is the particular realization under consideration, then the statement may be rewritten "there is an $\mathrm{x}$ such that $\mathrm{x}$ is John and $\mathrm{x}$ writes." As it pertains to the ethical principle of beneficence, the negated form "not doing harm," is the negative construction that may be thought of as that which results from a metaphoric rarefaction in which there is stepping back to allow for the more general form. In so doing, "doing no is it true that doing good is equivalent with not doing 
harm? Yes. Conversely, is it true that not doing harm is equivalent to doing good? It is possible yes and possible no; but not necessarily so, which makes the statement contingent.

A statement is considered to be contingent if it is both possible that the statement is true and possible that it be false [13] (Cook, 2009). Therefore, this first relational comparison reveals an asymmetrical relation between both beneficence and nonmaleficence such that necessarily if beneficence, then nonmaleficence, yet it is possible if nonmaleficence, then beneficence AND its possible if nonmaleficence, then not beneficence as well. That is, to say, the conditionalization of beneficience to nonmaleficence is one of necessity whereas that of nonmaleficence to beneficence is one of contingency. If $x$ 's doing good for $y$ allows $y$ to do good for $z$, then is it true that Bxz? That is, if Bxy and Byz, then Bxz true? Yes, so B (beneficence-doing good) and N (nonmaleficence-not doing harm) as they are in relation to one another are so transitively.

There are other such relations concerning $B$ and $N$ as well, which model the manner in which a relation may be used to determine the nature of the status. In addition, the relationship between intent and deception may be analyzed in much the same fashion as beneficence/nonmaleficence was, which should assist in uncovering the semantics of the connection that is shared. Let us suppose for our hypothetical scenario there exist two distinct individuals. Each of them is named Frank Frauder. One Frank has a middle initial D., and the other has $\mathrm{E}$ as an initial. Both are males, similar appearance but not identical. Let's assume they live in the same state, perhaps different towns, and unique addresses with different phone numbers, too. However, they were born on the same day, or work in the same company, which will make it interesting.

Although unlikely, these facts are all theoretically possible, yet are not as important as the perspectives from which we will attempt to make a determination. We will now begin with this as the base case and assume from the following different perspectives: 1 ) that from fraud being known to occur but not committing it, and 2) that from fraud being known to occur and being the one committing it. The perspective of the one committing it will be divided into wittingly (i.e., knowing actions is fraud/unethical) and unwittingly (i.e., not aware action unethical) committing fraud. Knowing that fraud occurred not having been the one to commit it. Thus, the question of intent concerns how best to phrase the action that resulted in the fraud: as an affirmation or denial. Is it writing or signing the name of another, or not signing one's name, that most adequately captures what transpires?

As the intention before it was done, once completed, however, the action(s) successfully carried out become the deception. The only difference between the two aspects relates to the ontological status. In other words, the ontological status pre-commission distinguishes intention from postcommission at which point the intention becomes manifest as the deception itself.

While it is possible for the action to be carried out yet be unsuccessful despite having the intention, it is before being carried out and success in having been carried out that are the main concerns. From these two outcomes as perspectives, all four possibilities may be determined accordingly. Before being carried out delineates or demarcates pure intention from anything else, which may not be verifiable; if the intention is successfully carried out, however, then we know that deception occurred, and there were consequences as well as intention. Carried out unsuccessfully implies that the act itself is what matters whether intention preceded it or not. Success pertains to intention and action; deception to intention and action.

\subsection{Relevant Dichotomies}

In consideration of what we know regarding the notion of fraud based on the definition previously given, the intent to deceive stipulation resulting in the two aspects of intention and deception as criteria for determination may be understood through the manner in which they are related to one another. As it currently stands, however, it seems as though the definition of fraud stipulating intention to deceive may be incorrect.

In an effort to determine whether fraud, as defined, will hold, the nature of the relationship between intention and deception must be thoroughly vetted. We must ascertain as much relevant information about these two aspects as possible. Given the information will be pertinent to either one 
or the other if the two aspects are not identical, it is recommended that the approach to this task be by analyzing for what I have termed the "relevant dichotomies." Relevant Dichotomies may be understood as opposing pairs of qualities, properties, or characteristics that either the presence or absence of which allows for their classification into groups according to similarities and differences. In the case of intention and deception, the relevant dichotomies concern the binary relation each aspect as object bears on the other.

For example, let us suppose that we have just thrown into the air and let fall as they may two or more coins. I claim that, through relevant dichotomies, it is possible to separate the coins with ease. One relevant dichotomy would be the color. That is, while no coin may be a match, any of the coins may be split into groups of those that are either silver or near-brown. Another relevant dichotomy could be whether the coins are showing a particular side of themselves (e.g., heads). In this fashion, one could continue to identify other relevant dichotomies that would eventually result in all remaining coins being categorized.

There will be readers who swiftly point out that a coin could have landed on its edge in theory, which would result in a trichotomy: heads, tails, and edge. These individuals would wonder how this would be possible to reconcile since there are three outcomes possible and not two. Please note that purpose of selection of categorization was embedded in the name. Most crucial is the ability to make the process simplistic. Thus, the requirement of both relevance and dichotomization at each step achieves simplicity.

In the rare case that among tossed coins a coin is found to be standing on its edge, then neither head side nor tail side shows. Therefore, the relevant dichotomy would be showing sides either yes, or no. Then, the sides showing group undergoes dichotomization into heads or tails, which results in the three groups. While it may have been just as easy to trichotomize in this case, the point may have been missed by implementing dichotomization. Instead of three categories, in the event that there were ten or one-hundred different groups that could be formed, it would be readily understood how quickly -otomizing immediately into the however many categories encountered might overwhelm by becoming unnecessarily difficult. There is a distinction between taking additional time and being difficult: often, I would argue the way to discern the two would be that if a procedure takes less time to complete, but results in a higher chance of error, then that procedure would be labeled more difficult.

The approach for dichotomization is general to specific. Itt proceeds in a logically structured manner so as to minimize error despite taking as long or longer to complete. Nonetheless, because dichotomization requires only two categories, whether compared to trichotomizing, or more, I contend that it will always be more straightforward and faster to look upon a pile of items for rapid assessment - be the items coins dropped or otherwise - and notice a dichotomy (2) than a trichotomy (3) or higher split. In this particular case, this allows one to separate coins on their sides from those that landed on the edge-thickness almost instinctively and more rapidly without taking much more time to complete the dichotomization of the coins showing sides. Moreover, if such a procedure were carried out iteratively with the prior round's results serving as the basis for subsequent ones in a recursive process, completing a relevant dichotomy assessment would result in more easily managed, less error-prone, and finely categorized groupings.

\section{Dimensional Properties of Fraud}

The following propositions about aspects of fraud are crucial for understanding:

- In the event of success in the core aspect of intention, there would also be a success in deception (i.e., if the intention to forge a check exists and is carried out successfully (i.e., falsify a signature), then deception will have occurred).

- Additionally, were an individual to have failed in their attempt at the intention, the failure does not alter the fact that deception was the intent.

The potentially relevant dichotomies for intention and deception involve several properties and dimensions concerning the manner in which each relates to the other and to itself. Such dimensional 
properties include the ontological, the temporal, and those related to achievement. For instance, if $\mathrm{x}$ intends to deceive, then the following may be inferred about both intention and deception: The intention exists before the deception, and the deception exists after the intention.

We may also consider another relevant dichotomy, which concerns deception as there exists no way of realizing achievement directly; deception may only be considered successful indirectly through the achievement of intent when present. Thus, whether the intention was successful is the focus and what being successful means in the case of deception concerns us. Important to note is that, on its own, as an intangible, intention itself gives rise to the actions that result in the object of the original intention, which preceded them. Conversely, the perspective may be altered such that one could directly address whether the deception is successful and what that means for the intention when present. Such an alteration in viewpoint may provide valuable insight that would have otherwise been missed.

\section{Achievement Reveals Status of Intention}

Evaluation of the success or failure of fraudulent acts as a relevant dichotomy relates to both intention and deception. Upon evaluation, can any intention be said successful if it does not exist? No, it cannot. Thus, the intention must exist. Nonetheless, can deception be considered successful on evaluation if the intention to deceive does not exist? Yes. In other words, it is possible that someone may be deceived by another's actions without the existence of the intent to deceive.

For instance, on several occasions, by pure happenstance, the color and clothing I have worn resembled that of staff in a store to those failing to pay close attention. As a result, questions were asked of me that I was unable to answer. Upon my notification, the person who asked me replied "Oh, I'm sorry! I thought you worked here." It seems as though people were deceived into thinking I was someone I was not without my intention to deceive (i.e., commit fraud). So, this is a crucial piece of information that reveals something about the nature of deceit and the role that intentions, acts, and achievement play, if any, in their success.

If an intention to deceive is to be claimed successful, then it must itself exist. Also, because the intention is to deceive, a successful intention implies that deception was achieved. Nonetheless, even if the intention were not successful, then it would still have to exist; although, the deception would also be unsuccessful. It appears as though if the intention exists, then either it is successful or unsuccessful.

If the intent is successful, the deception is successful; if unsuccessful, then deception is as well. If the intention exists, then either deception is successful or unsuccessful. Therefore, since the intention must exist regardless of the success or failure of deception, the existence of intention cannot determine whether or not deception succeeds. It may be concluded that successful deception can occur independently of the existence of any intention to deceive. Additional questions then would include, if the deception were successful based on the fact that someone was deceived (e.g., the customer was wrong about me being an employee as a passive mistake without my intention or action), then is there still a case of fraud to consider? If so, then why might be worthwhile to ask.

Now that we have critiqued the conditionalized statement from left to right, we will consider the opposite direction for consistency. The converse line of reasoning begins with whether or not deception can be successful without the corresponding success of the intent. That is if the intent to deceive requires intention and deception if deception succeeds, can we determine anything about intention?

If deception succeeds, then it occurred. However, if the deception occurred (e.g., someone was under the impression of something about which he or she were wrong), then is it the case that an intention to deceive existed? Not necessarily, as the mistaken employee example highlights. The intention may or may not have existed prior to the occurrence of deception. Also, if the intention existed, then it may or may not have succeeded. Thus, since success in deception may or may not have been preceded by an intention to deceive that existed, it cannot be considered a determiner of the success of the deception. Also, even if there exists an intention, which was successful, success in deception cannot be said to depend on or be a result of, the success of the intention to deceive. 
The issue that arises is deception, when successful, exists; when it is unsuccessful, it does not exist. There is no way for deception to exist but be unsuccessful like intention. It is in this respect that the status of intention may be said higher than that of deception. Once the intention is reduced to the level of deception by being executed, it becomes equivalent with deception through its demotion such that it may no longer exist independent of success or failure.

There is something fascinating about the nature of intention and that of deceit, which merits mentioning. When comparing among derivations the verbs and noun word-forms, grammatically, verbs give rise to nouns in use. For instance, if I sing, then singing results. Also, if I think, thinking results. So, whether physical like singing or cognitive like thinking, performance of the action necessarily results in the action occurring. Furthermore, the occurrence of the action is by the one who performs the noun. That notwithstanding, though very unusual in the case of fraud, because the occurrence of deception (the noun) is not guaranteed by, or as the result of, the one who performs the action with or without an intent to deceive (the verb), there cannot be said to exist a causal relationship such that the performance of the verb as cause bears this relation to the occurrence of the noun that is the effect. Thus, it appears as though intention, which becomes linked to deception through the performance of an action, sometimes is inextricable from deception when it is successful, yet other times is irrelevant. Such an extremely complicated relationship will likely require a particular framework to characterize it better. Next, we consider Supervenience Theory as a possible contender and explain it will not work.

\subsection{Supervenience as a Framework for Comprehension of Aspects of Intent of Deception}

Having established the relationship as mentioned earlier regarding intent and deception, it may be beneficial to note that concerning falsification with respect to the classification status of fraud, as a tool for analyzing the concept of supervenience could potentially provide a framework for understanding. Supervenience theory refers to the relation between two sets of properties, one of which seemingly emerges from another that is more basic [19] (Mclaughlin and Barrett, 2011). Although more basic can be understood to mean core, as it applies to the present topic, I argue the following: It is not the case that the designation of fraud as a status for misconduct supervenes on the status of intent and deception being considered.

The basis for claiming fraud does not supervene on intent and deception is that a difference in fraud status (i.e., guilty/not, or occurred/did not) does not require a difference in the status of either intention or deception. That is, to say, according to supervenience theory, A-properties supervene on B-properties if and only if a difference in A-properties cannot come about without a corresponding difference in B-properties - or, equivalently, if and only if exact similarity with respect to B-properties guarantees exact similarity with respect to A-properties [19].

For fraud, as it may be said to supervene on intention and deception, if the difference in the property going from not being fraud to being fraud is determined, then there must be a corresponding difference going from not having the intent to deceive to having the intent to deceive. However, since it has been demonstrated that having the intent to deceive does not guarantee that what was done was successful in deceiving, then it is possible for there to exist no difference in the outcome of an action or behavior when it is done when not having the intent to deceive and while having the intent to do so. That is, to say, it is possible that if one has the intent to deceive, performs actions to achieve that end having the intent, and as a result of the actions performed no deceit occurs, then no success in deception from actions performed having the intent would imply all three are distinct from one another.

Recall, the absence of a corresponding is what it takes to discern that which supervenes from that which does not. Manipulating the variables individually while holding all else to determine possible outcomes is the experiment. It is possible that deception occurs, but not as a result of the actions performed; in this case, the intent to deceive would be successful through no fault of the act performed regardless of the intention. Likewise, deception could be both successful, and the result of actions performed, yet not be the deception intended. The main point is that although these may cooccur, an intent to deceive, actions performed intended to achieve such an end, and what the outcome 
will be are not necessarily connected causally. But, if so, then supervenience as a framework for understanding will not hold. Since there appear to be so many fluctuations in outcome and variables that the only consistent thing about the aspects and relations concerning fraud designation seems to be that it depends on the particular combination of variables as context. Thus, in abandoning supervenience as a possibility, contextualism is the next consideration for a framework for understanding.

\subsection{Contextualism as a Potential Framework for Understanding}

Contextualism is the view that the overall meaning is a function of more than just the meaning of constituent parts and the manner in which those constituents are combined to form a complex expression - in particular, the context within which that expression occurs contributes to its meaning [13] (Cook 2009). As a philosophical framework for understanding, contextualism has a lexical counterpart in the word deictic. Contextualism is to an expression as deictic is to a word. Thus, the adjective deictic describes a word whose meaning is dependent upon the context within which it is being used [18] (Oxford 2017a). Both contextualism and deictic bear a relation to one another and themselves, the appreciation of which I claim has the potential to assist in understanding falsification conceptually. Furthermore, if the meaning of an expression is a function of more than just the meaning of its proper parts (semantics) and the manner in which those parts combine (syntactics), then as the context within which the expression occurs, which comprises the semantico-syntactic understanding, what additional non-semantico-syntactic dimensions comprise the context? Moreover, would a determination regarding the additional things comprising the context help us progress beyond the current state of understanding in which we are? Some non-semantico-syntactic aspects of context may include the ontological and the locative. While these aspects are neither explicitly stated nor directly related to the meaning of the words or expression per se, their ontological or locative status nonetheless can influence the overall meaning of an expression. If the context within which the meaning of an expression is being determined hinges on the ontological status or locative status of an aspect not explicitly stated, then the overall meaning of an expression may be impacted.

For instance, the statement: the person in front of you now standing on the platform has given birth twice. The meaning of the expression varies depending on the person. However, if there is no person (ontological status is nonexistent or false) or there is a person, but not in front of you (locative status is not where you said when you said it/false), then the meaning is impacted. Given the inconsistency that has been encountered based on the definition of fraud that required the intent to deceive, how do we reconcile what was deduced to adequately define fraud? That is, if the following statements are true, then:

- Fraud comprises the intent to deceive

- The intent to deceive consists of two aspects: intention and deception

- Using relevant dichotomies to describe the properties of the relation of both to one another and each to themselves it was shown that the aspects are consistent and possible together but not necessarily so

- The intent to deceive either may exist or may not exist (i.e., is contingent) in instances of successful deception

- If the intention is to deceive does exist, then either it may be successful, or unsuccessful

- If the intention to deceive is successful, then both the intention is successful, and the deception is successful.

Upon analysis success in deception appears to occur independently of the existence of any intent to deceive. Whether the intent was achieved (i.e., deception occurred), or not should not be what determines guilt in instances of fraud; that there was intent to deceive is paramount. Furthermore, although without the aspect of intention there can be no fraud, given successful cases of fraud result in the occurrence of deception, whether the intent was real or merely perceived from the perspective of someone who claims to have been deceived, the aspect of deception must have been the intent. 
While resembling the form of an inference to the best explanation given available evidence, the informal fallacy that is applicable in this situation concerning fraud is also known as affirming the consequent [13] (Cook 2009). When the consequent of a conditional statement, which is the latter half preceded by "then," fallaciously is affirmed, the error is made in concluding that since the consequent is the only observation that was made of the conditional, the antecedent is responsible.

In the case of the definition of fraud, the intention precedes the deception. Clearly, this must be true since one cannot claim an intention to deceive following the occurrence of the deception. Furthermore, it is not possible to simultaneously have intended that which occurs as it is occurring. Thus, the conditional formulated from the definition may be stated as "if intention, then deception." However, if someone is deceived, then they incorrectly affirm the deception based on the formulated conditional relationship by assuming that there had to be the intention for it to occur. Of course, this logical fallacy is the result of faulty logic and the creation of a false causal relationship between intent and deception. Thus, deception can occur without intent the same way that intent can occur without deception.

\subsection{Hypothesis Concerning De Facto Fraud Classification in the Literature}

Despite the technical definition of the term fraud according to which each constituent of FFP would qualify as an instance, it appears as though the classification of fraud has been applied to certain types of traditional misconduct to the exclusion of others, which results in the formation of a de facto group under the rubric of fraud. The forms of misconduct classified as members of this de facto group under fraud include both fabrication and falsification (Stroebe, Postmes, and Spears 2012).

An analysis of the de facto forms (i.e., fabrication and falsification) categorized as fraud in the literature was the starting point for obtaining insight into the basis for such classification of misconduct. When characteristics of de facto types are compared to that which is not included among them as fraud (i.e., plagiarism), the fifindings lead me to hypothesize that several perspectives could have been used to make the distinction. Of the forms comprising the de facto fraud group, there is at least one characteristic or property they share that the other type of misconduct that is excluded from the group lacks. That characteristic, I argue, is the de facto forms being, or directly resulting, from the actions or behavior of individuals that occurs while performing research.

While it could be maintained that such a characteristic may be significant for multiple reasons from any one perspective, it may also be for multiple reasons from a variety of perspectives. Regardless of the perspective, however, the ability to utilize this quality to distinguish particular types of misconduct from one another is paramount. Also, an awareness of the characteristic of the occurrence of an act of misconduct may also be used as a basis according to which one may determine the level of perceived gravity. That is, de facto fraud can be deemed more severe than forms that are not included in the de facto group. Alternatively, those forms of misconduct classified as a fraud not only characteristically occur during the conduct of research, they also directly lead to the distortion of knowledge. In fact, according to the distortion of knowledge criterion as a characteristic to classify the de facto group members, the distinction could be seen as even more pronounced than with misconduct occurring solely while research is being done.

Based on the analysis of the characteristics of the fraud, my hypothesis is that the grouping suggests that the determining factors were either "when" or "what" has occurred. Either factor may be equally useful in determining a classification system for misconduct as fraud. Furthermore, if correct, then the absence of these aspects should explain the exclusion of other forms of misconduct. Thus, although plagiarism as a type of misconduct may be considered as deceitful, it was not classified as fraud. I claim that plagiarism was excluded since misappropriating the work of another and presenting it as one's own neither results from actions that occurred during the conduct of research, nor directly leads to distortion of knowledge (i.e., concerning the content of the work itself), which maintains consistency with the aspects of when and what failing to be satisfied when not included as a form of de facto fraud according to such a method of determination. 
The decision to address falsification as a type of fraud under research misconduct in the next paper was partly based on falsification being considered one of the most severe types of misconduct [20]. In consideration of the serious nature of the consequences that may potentially result from involvement in suspected cases of falsification as misconduct, I claim that further elaboration and refinement of the definition of falsification as misconduct under the rubric of fraud is warranted and would likely reduce the number of new allegations and convictions. Through the provision of such transparency as refinement in definition would bring, the resultant clarification would not only contribute to the deterrence of misinformed allegations but also reduce the potential for careerending false convictions for individuals unjustly accused. It is for these reasons that a conscious decision has been made to focus on the definition of falsification in the next installment.

Funding: This research received no external funding.

Conflicts of Interest: The authors declare no conflict of interest.

\section{References}

1. ORI Data | ORI - The Office of Research Integrity.

2. Merriam-Webster. Definition of Allege, 2017.

3. Turvey, B.E.; Savino, J.O.; Baeza, J.J. False Allegations: Investigative and Forensic Issues; 2017.

4. $\quad$ Neiman, T.; Loewenstein, Y.; Shteingart, H. The Role of First Impression in Operant Learning. Journal of Experimental Psychology: General 2013, 142, 476-488.

5. Behrens, L.; Rosen, L. A Sequence for Academic Writing. Pearson Education, 2017.

6. Huang, I.N.; Tomasini, J.; Nikl, L. The Primacy and Recency Effects in Successive Single-Trial Immediate Free Recall. Journal of General Psychology 1977, 97, 157-165.

7. Wu, Y.; Qian, Z.; Riguidel, M.; Chen, G. CogTag: Cognitive tags framework for new generation Internet beyond IP \& MPLS. Retrieved 12, 2013.

8. Pediaditakis, N. The association between major mental disorders and geniuses. The Psychiatric Times 2014, 31, 1-4.

9. Morrison, A.B.; Conway, A.R.; Chein, J. Primacy and recency effects as indices of the focus of attention. Frontiers in. Human Neuroscience 2014, 8, 6-6.

10. Michalek, A.M. "The costs and underappreciated consequences of research misconduct: A case study." In: PLoS. Medicine 2010, 7, 1000318.

11. Hey, E.I. "Mis-investigating alleged research misconduct can cause widespread, unpredictable damage."

In. JRSM 2010, 103, 133.

12. Cook, R.T. A Dictionary of Philosophical Logic; Edinburgh University Press, 2009.

13. Fellbaum, C. WordNet: An Electronic Lexical Database. Electronic, 1998. www.easydefine.com(visited

14. Martella, R.C. Understanding and interpreting; 2013; p. 1.

15. Browne, M.N.; Keeley, S.M. Asking the Right Questions: A Guide to Critical Thinking. Pearson Education, 2017.

16. Orilia, F.; Swoyer, C. Properties. The Stanford Encyclopedia of Philosophy, 2017.

17. New Oxford American Dictionary. - 2017.

18. McLaughlin, B.; Bennett, K. Supervenience. Metaphysics Research Lab, Stanford 
University, 2018.

19. George, S.L. "Research misconduct and data fraud in clinical trials: Prevalence and ca $\quad$ causal factors." In. International Journal of Clinical Oncology 2016, 21, 15-21. 